\title{
Der schwierige Weg von guter Qualität zu besserer Vergütung
}

\section{Peter Berchtolda ${ }^{a}$ Oliver Reich ${ }^{b}$}

a PD Dr. med., Präsident fmc - Schweizer Forum für Integrierte Versorgung

${ }^{b}$ Dr. phil., Leiter Gesundheitswissenschaften, Helsana Gruppe; Vorstandsmitglied fmc

\author{
Gleich wer eine medizinische Leistung erbringt, ob Arzt, Pflegefachperson, Apo- \\ theker oder Physiotherapeutin - neben der eigentlichen Leistung sollte auch das \\ vergütet werden, was für den Patienten oder die Patientin im Zentrum steht: die \\ Qualität und das Ergebnis der Behandlung oder Betreuung. Oder in einem Wort: \\ die Performance.
}

So einleuchtend das erscheint, so komplex ist die Umsetzung. Nicht für die eigentliche Leistung, dort ist die Sache ziemlich klar: Es braucht eine Leistungsbeschreibung, zum Beispiel eine TARMED-Position oder Fallpauschale, sowie einen Preis in Form eines Taxpunktwerts oder einer Baserate.

Bei der Performance sieht die Sache deutlich anders aus. Bis heute gibt es nur sehr begrenzte Möglichkeiten, die Qualitätskomponenten einer Leistung in Franken und Rappen zu übersetzen. Und dies, obwohl in den vergangenen Jahren viel in Qualitätsindikatoren investiert wurde und diese in unzähligen Studien wissenschaftlich evaluiert wurden. In einen Satz gefasst: Der exakten Bestimmbarkeit von Leistungen und Preisen kann (zumindest heute) kein annähernd ausreichendes Bewertungssystem zu Qualität und Performance gegenübergestellt werden. Damit nicht genug: Viele der bisherigen Performanceorientierten Vergütungsmodelle sind bei wissen-

\section{«Plus d'utilité pour les patients et une meilleure}

\section{rémunération par l'intégration»}

Les effets indésirables (p. ex. prise en compte disproportionnée d'indicateurs «rentables», mesures biaisées) des modèles de rémunération basés sur les performances sont fréquents. Or les tarifs à la prestation et les forfaits par cas actuellement en vigueur ont également un impact défavorable (p. ex. augmentation du volume de prestations, doublons). Les modèles de rémunération basés sur les performances doivent donc être conçus et appliqués de manière appropriée. Pour cela, il s'agit de mesurer l'ensemble du processus de prise en charge et de traitement et pas uniquement la prestation individuelle. La performance doit faire référence à l'ensemble du collectif de patients et pas à un cas particulier. La Suisse est particulièrement adaptée à ce type de modèle, car il existe depuis 20 ans des modèles de rémunération pour les médecins ou les réseaux de soins qui se réfèrent à un collectif de fournisseurs de prestations et de patients. La coresponsabilité budgétaire négociée entre les réseaux et les assureurs permet d'être plus conscient de l'ensemble du processus et d'y intégrer systématiquement les fournisseurs de prestations. schaftlichen Evaluationen mehr oder weniger durchgefallen. Vor allem deshalb, weil sie neben den beabsichtigten Wirkungen auch unerwünschte Nebenwirkungen zeigten. Leistungserbringer tendierten beispielsweise dazu, Performance-relevanten Indikatoren überproportional Beachtung zu schenken und dafür andere Bedürfnisse der Patienten zu vernachlässigen oder - im schlimmsten Fall - Qualitäts-

Bis heute gibt es nur sehr begrenzte Möglichkeiten, die Qualitätskomponenten einer Leistung in Franken und Rappen zu übersetzen.

messungen zu manipulieren, um die eigene Vergütung zu maximieren [1].

Zudem fokussiert die Mehrheit der heutigen Modelle auf einzelne Leistungserbringer (anstelle aller am Behandlungsprozess Beteiligten) und festigt damit die sektorale Behandlung, statt die Integration zu fördern. Ein weiterer Einwand ist das drohende "Rosinenpicken", wenn Leistungserbringer also nur noch leicht therapierbare Patienten ohne komplexere Probleme betreuen, weil so die Performance-Ziele (und damit eine höhere Vergütung) einfacher zu erreichen sind. "Gaming the system" lautet der wenig schmeichelhafte Begriff dafür.

Vor dem Hintergrund solcher Erfahrungen drängt sich also die Frage auf: Sind Performance-orientierte Vergütungsmodelle grundsätzlich abzulehnen? Wohl kaum, denn unerwünschte Nebenwirkungen hat jedes Anreiz- oder Vergütungssystem, auch die derzeit wichtigsten Vergütungsformen wie Einzelleistungs- bzw. Zeittarif oder Fallpauschalen. Gerade aus Patientenperspektive gibt es eine ganze Liste an Vorbehalten gegenüber diesen Vergütungsformen. Als augenfäl- 


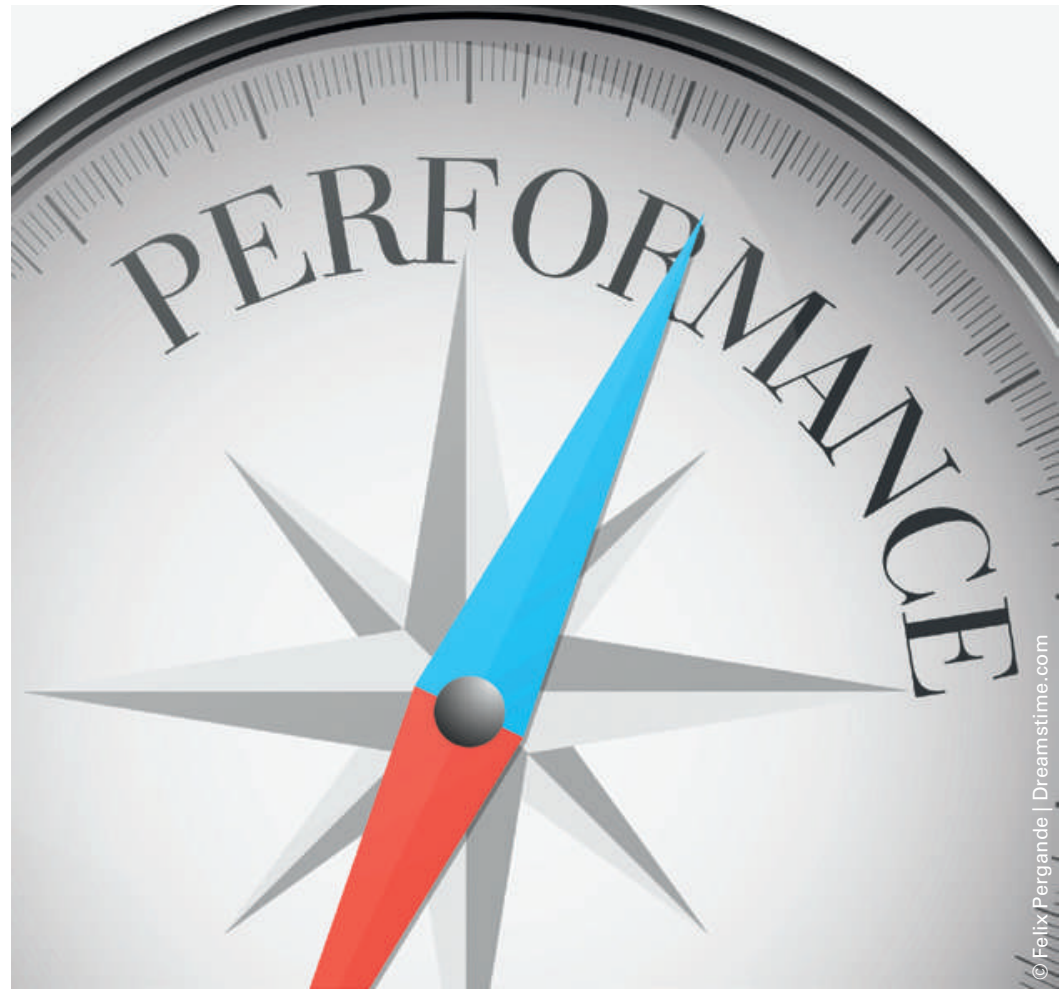

Performance nimmt immer auch die gesamten Behandlungs- und Betreuungsprozesse in den Blick - und nicht nur den einzelnen Spitalaufenthalt oder die Betreuung durch die einzelne Fachperson.
Integrierte Versorgung benötigt eben auch integrierte Vergütungsmodelle.

Gerade dafür sind Performance-orientierte Vergütungsmodelle besonders geeignet. Denn Performance nimmt immer auch die gesamten Behandlungs- und Betreuungsprozesse in den Blick. Und das ist es, was für $\mathrm{Pa}$ tienten, besonders chronisch kranke, entscheidend ist und nicht der einzelne Spitalaufenthalt oder die Betreuung durch die einzelne Fachperson.

Bewerten lässt sich das zum Beispiel mit longitudinalen Performance-Indikatoren, die wenn immer möglich auch für andere Zwecke genutzt werden (Krankheits-Register, Administration). Damit kann der Zusatzaufwand der Performance-Messungen möglichst tief gehalten werden. Gerade administrative Daten der Krankenversicherer bieten eine Fülle von Performance-relevanten Parametern. Die vor kurzem vom Institut für Hausarztmedizin der Universität Zürich, dem Kantonsspital St. Gallen und der Helsana veröffentlichte Arbeit, in der die Einhaltung von Guidelines bei Diabetes-Patienten überprüft wurde, zeigt das beispielhaft [3].

Aber sind solche Performance-orientierte Vergütungsformen in der Schweiz überhaupt möglich? Und falls ja: Wie muss man sich diese vorstellen? Was vielen nicht bewusst ist: In der Schweiz gibt es schon länger bedeutsame Erfahrungen mit Vergütungsmodellen, die sich auf ein Kollektiv von Leistungserbringern und Patienten beziehen - nämlich die Budgetmitverantwortung von Ärztenetzen. Auch wenn sie in der Ärzteschaft immer noch umstritten ist, haben sie fast alle Ärztenetze seit Jahren in ihren Verträgen mit den Krankenversicherern vereinbart.

Zur Erinnerung: Budgetmitverantwortung meint die institutionelle Mit-Verantwortung des Ärztenetzes genausweitungen verursachen und den Patienten (zu) viele Doppelspurigkeiten zumuten.

"There is a case for including pay for performance as part of physicians' payment, not least because of the problems associated with all other payment systems»: Mit dieser Folgerung schliesst ein vor kurzem im New England Journal of Medicine erschienener Report, einer der renommiertesten Fachzeitschriften und nicht dafür bekannt, gegen die Medizin und die Mediziner zu polemisieren [2].

Diese grundsätzliche Kritik an allen «anderen» Vergütungsformen darf nicht erstaunen. Denn die Medizin hat sich seit der TARMED- oder DRG-Einführung stark gewandelt. Folglich liegt es auf der Hand, dass auch unsere aktuellen Vergütungsformen je länger, desto weniger mit den stetig steigenden Anforderungen Schritt halten. Optionenvielfalt und Komplexität der Behandlungen, Interprofessionalität und Interdependenz der Betreuung verlangen mehr Integration sowohl in der Versorgung wie auch bei der Vergütung.

\section{Performance nimmt immer auch die gesamten} Behandlungs- und Betreuungsprozesse in den Blick.

(und nicht des einzelnen Arztes) für eine bestimmte Patientengruppe und zwar bis zu einer festgelegten Risikolimite bei vereinbarten Kostenzielen. Wesentlich ist dabei, dass diese Budgets Parallelrechnungen darstellen, in der alle von den Versicherern für das jeweilige Patientenkollektiv bezahlten Rechnungen verbucht werden (niedergelassene Ärzte, Spital, Apotheke, Spitex, Therapien, stationäre Rehabilitation etc.).

Die Rechnungsstellung und die Vergütung der Leistungserbringer erfolgen im jeweils gültigen Tarifsystem, also TARMED, DRG oder Spitex-Tarif. Berechnungsgrundlage der Budgets ist eine Kopfpauschale (Capitation), d.h. jener Betrag (ambulant und stationär), den ein einzelner Versicherter im kommenden 
Jahr in Abhängigkeit von Alter, Geschlecht, Wohnort und Gesundheitszustand voraussichtlich benötigen wird. Die Summe der Kopfpauschalen ergibt dann das Budget für das Ärztenetz [4].

\section{Aber sind solche Performance-orientierte Vergütungsformen in der Schweiz überhaupt möglich?}

Die Budgetmitverantwortung schärft also den Blick und das Bewusstsein für den gesamten Behandlungsund Betreuungsprozess und damit das Zusammenspiel aller Leistungserbringer - oder auf einen Nenner gebracht: die konsequente Integration der Versorgung. Folglich lässt sich die Budgetmitverantwortung, die primär auf Einsparziele fokussiert, ideal mit der Performance-orientierten Vergütung ergänzen. Zumal das Krankenversicherungsgesetz es zulässt, die Budgetberechnung und damit die Mitverantwortung der Leistungserbringer um Performance-Kriterien zu erweitern. Selbstverständlich sollten in solchen Performance-orientierten Verträgen alle beteiligten Leistungserbringer - niedergelassene Ärzte, stationäre Einrichtungen, Langzeitpflege, Apotheker, Therapeuten - eingeschlossen sein. Diese müssten dann gemeinsam klären, welche Performance sie ihren Patientinnen und Patienten gemeinsam bieten wollen.

\section{Referenzen}

1 Eijkenaar F, Emmert M, Scheppach M, Schöffski O. Effects of pay for performance in health care: A systematic review of systematic reviews. Health Policy 2013;110:115-30.

2 Roland M, Campbell S. Successes and Failures of Pay for Performance in the United Kingdom. N Engl J Med 2014;370:1944-9.

3 Huber CA, Brändle M, Rapold R, Reich O, Rosemann T. A set of four simple performance measures reflecting adherence to guidelines predicts hospitalization: a claims-based cohort study of patients with diabetes. Patient Preference and Adherence 2016;10:223-31.

4 Berchtold P. Budgetmitverantwortung in der integrierten Versorgung. Schweiz. Ärztezeitung. 2010;91:18.

\section{Nationales Symposium Integrierte}

\section{Versorgung, 15. Juni 2016, Kursaal Bern}

Das Nationale Symposium 2016 des fmc - Schweizer Forum für Integrierte Versorgung beleuchtet unter dem Titel «Mehr Patientennutzen: bessere Vergütung durch Integration" das Zusammenspiel zwischen Integration, Qualität und Vergütung Renommierte Experten aus dem In- und Ausland liefern Impulse, wie die Performance der Integrierten Versorgung sichtbar, messbar und erlebbar gemacht werden kann. Ausserdem können die Teilnehmenden das Thema in moderierten Tischgesprächen und in Workshops vertiefen. Und sie erfahren erste Resultate der schweizweiten Erhebung zur Integrierten Versorgung, die das fmc gemeinsam mit der Universität Lausanne und dem Gesundheitsobservatorium durchführte. Weitere Informationen zum fmc-Symposium 2016 finden Sie auf www.fmc.ch.

Das Symposium 2016 wird vom Schweizerischen Institut für ärztliche Weiter- und Fortbildung (SIWF) mit 5 Credits anerkannt. 